\title{
Factores que Determinan el Diseño de Servicios Diferenciadores en Empresas del Sector de Suministros Industriales: Caso GRAINGER
}

\section{Factors Determining the Design of Differentiating Services in Companies in the Industrial Supplies Sector: the GRAINGER Case}

Francisco Vergara-Streinesberger*

Institución Universitaria CEIPA - Colombia

ORCID iD: https://orcid.org/0000-0001-7402-1144

francisco.vergara@estreico.com

\section{Yarelis Aycardi-Vitola}

Universidad Tecnológica de Bolívar - Colombia

ORCID iD: https://orcid.org/0000-0002-6001-7491

yaycardi@utb.edu.co

\section{Katlhin Mendoza-Merchán}

Universidad Tecnológica de Bolívar - Colombia

kmendoza@utb.edu.co

* Autor a quien debe ser dirigida la correspondencia
Fecha de recepción: 21/06/2020

Fecha de evaluación: 29/06/2020

Fecha de aceptación: 30/07/2020

Cómo citar: Vergara-Streinesberger, F., Aycardi-Vitola, Y., \& Mendoza-Merchán, K. (2020). Factores que Determinan el Diseño de Servicios Diferenciadores en Empresas del Sector de Suministros Industriales: Caso GRAINGER. Revista Cientifica Anfibios, 3(2), 37-53. https://doi.org/10.37979/afb.2020v3n2.72

\section{Resumen}

Hoy en día las empresas deben diseñar estrategias de manera continua y constante donde les permita ser más atractivas para los clientes frente al mercado, es por esto que esta investigación se buscó analizar en primera instancia las necesidades de los clientes a partir de diversas herramientas, con las cuales se logró realizar una compilación de las necesidades básicas, requerimientos y expectativas de los clientes, y de esta forma se pudo identificar a través de dichas variables cuales son los factores que determinan el diseño de un servicio diferenciador para empresas del sector de suministros industriales. A medida que pasa el tiempo, los mercados y clientes son y serán cada vez más demandantes, es por ello que las organizaciones deben esmerarse y esforzarse no solo para brindar servicio si no en marcar diferencia frente a sus competidores a través de un punto clave y estratégico que es conocer primeramente la voz del cliente la cual puede hacerse a través de encuestas y/o entrevistas con preguntas sencillas y todo enfocado a cuáles son sus expectativas que ellos como clientes potenciales esperan y desean. En definitiva, el poder conocer la Voz del cliente permitirá a la generación de valor para la empresa y que este tenga un servicio diferenciador donde todos sus clientes estén dispuestos a pagar.

\section{Palabras clave:}

Estrategia; diseño de servicios; diseño organizacional; suministros industriales; servicio diferenciador.

\begin{abstract}
Nowadays companies facing the market must design strategies in a continuous and constant way where it allows them to be more attractive to customers, which is why this project aimed analyze customers' needs in a first basis, upon several tools, in which a compilation of the basic needs, requirements and expectations of customers was carried out, and in this way allowed to identify through these variables, which are the factors that determine a differentiating service design for industrial supplies sector companies. As time passes, the markets and customers are and will be increasingly demanding, which is why organizations must strive not only to provide service but to make a difference against their competitors through a key point and strategic which is to know firsthand the voice of the customer which can be done through
\end{abstract}


surveys and / or interviews with simple questions, all focused on what are their expectations that they as potential clients expect and want. In short, being able to know the Voice of the customer will allow value generation for the company and that it has a differentiating service where all its clients are willing to pay.

Keywords:

Strategy; service design; organizational design; industrial supplies; differentiating service.

\section{Introducción}

Las Organizaciones actualmente viven un momento en donde las exigencias de los clientes cada vez son mayores, es por esto que las empresas deben siempre trabajar en brindarles un valor agregado e innovador en el servicio que presta; donde ellos puedan notar el servicio exclusivo que tienen y puedan sentirse que exceden sus expectativas y su deseo como consumidor; permitiendo que los frentes competidores no absorban su mercado ya cubierto y ganado.

Teniendo en cuenta que el continuo desarrollo industrial que se ha estado enfrentando en este mundo globalizado, ha llevado a la apertura de nuevos competidores y a la aparición de productos y servicios a través de un valor agregado innovador que es un recurso estratégico clave para poder alcanzar una ventaja competitiva en el mercado, el sostenimiento económico y conduce a generar mayor y mejores utilidades, resaltando que las utilidades no son sostenibles si no hay una relación gana-gana con los clientes y en general con los grupos de interés.

Con base en los elementos anteriores, el problema que se plantea en esta investigación es que el objetivo de la diferenciación no solo es actuar conforme a las necesidades o expectativas dadas por los clientes sino es buscar sorprender a los clientes satisfaciéndolos con alguna característica o requerimiento que no han expresado. Las empresas siempre deben buscar nuevas formas de impactar a sus clientes de acuerdo a las necesidades que van evolucionando con el tiempo.

Es por ello que los clientes de las empresas del sector de suministros de equipos para mantenimien- to, reparación y operación industrial valoran mucho los plazos en las entregas de sus órdenes además de facilitar la distribución en canales de distribución diferente a los habituales, debido a que ellos requieren sus productos de manera inmediata una vez identifican la necesidad para reparar o hacer mantenimiento a sus plantas productivas, mineras, químicas, construcción, entre otras; y esto es de suma importancia para ellos ya que va ligado a las perdidas en merma y paradas; por no contar con sus productos en el menor tiempo posible, siendo este uno de los puntos claves en los que se deberían trabajar para tener ese valor diferenciador frente a la competencia.

Con base a lo anterior, se tomó como evidencia de la problemática que la empresa Grainger Colombia la cual, al momento del inicio de esta investigación (2016) estaba presentando quejas de parte de sus clientes en cuanto a el servicio en tiempo de entregas donde el $83 \%$ de sus clientes se sentían insatisfechos (datos tomados de los resultados de la encuesta de satisfacción y necesitaban que existiera un servicio diferenciador para que sus órdenes no fueran entregadas en un tiempo mayor a 24 horas, debido a que ellos lo necesitan con urgencia una vez que realizan la orden para solicitar sus productos. La tabla 1 muestra los resultados reportados por los clientes al momento de recibir el servicio, sobre una muestra de 428 clientes, Presentando diferencia por la competencia de este mismo sector donde los pedidos son entregados en el tiempo que dispone el cliente al momento de realizar la compra utilizando técnicas y estrategias para que él sea en el menor tiempo posible, tales competidores como Homecenter, metrópolis center, Herramienta \& seguridad, Meico entre otros.

Tabla 1. Datos de resultado de encuesta de servicio en Q4/2016

\begin{tabular}{ccc}
\hline \multicolumn{3}{c}{ En escala que tan satisfecho se siente con el servicio de Grainger. } \\
\hline Answer Options & Response Percent & Response Count \\
Satisfecho & $17,0 \%$ & 73 \\
Nada Satisfecho. & $83,0 \%$ & 355 \\
\hline \multicolumn{3}{c}{ Fuente: elaboración de los autores }
\end{tabular}


Es por ello, que el problema de investigación que se planteó es el bajo nivel de satisfacción de los clientes por el servicio prestado, lo cual constituye un reto estratégico para lograr la permanencia en el mercado

\section{Fundamento teórico}

Los negocios hoy en día son exitosos cuando satisfacen las necesidades de los clientes brindándole siempre un elemento diferenciador al servicio que ofrecen con el fin de enamorar a los clientes, atraerlos y fidelizarlos, podemos decir que, si una organización hoy en día no cuenta con este elemento clave e importante simplemente la coloca fuera de competencia, así mismo puede ocasionar la no generación de utilidades debido a que sus clientes no se sienten atraídos para permanecer comprando. Tal como plantea Lovelock (2004), las expectativas de las personas sobre los servicios están influenciadas por varios aspectos: sus experiencias previas como clientes, comentarios de otros clientes, necesidades personales, sector que presta el servicio, entre otros.

Actualmente la experiencia del cliente en un servicio prestado es una opción de posicionamiento estratégico en las organizaciones donde su mayor reto es convertirla en una mayor oportunidad para generar una ventaja competitiva, logrando así que este se convierta en ese elemento diferenciador ya que la clave está en conocer la voz del cliente vs la promesa de valor que tenga la organización con el fin de generar beneficios facilitadores que permitan ser impulso para el negocio.

La razón anterior, llevó a realizar esta investigación con el fin de identificar cuáles son los factores más relevantes que lleven a impulsar a las organizaciones a diseñar una característica y/o elemento diferenciador en su servicio prestado ya que debido a la gran cantidad de competidores que hoy en día hay en el mercado y a la alta exigencia de los clientes es un requisito que debe cumplir todas las organizaciones si quieren alcanzar el éxito y la sostenibilidad económica representando una oportunidad importante para adquirir una ventaja competitiva y así pueda abarcar una mayor participación de mercado.

Basándose en el texto "Bye, Bye, Marketing: Del poder del mercado al poder del consumidor" (Medina, 2010), se puede hacer un recorrido a través de la historia del marketing, con el cual se puede interpretar que desde el inicio de la historia el comercio ha desempeñado un papel fundamental en el desarrollo de la sociedad, a la hora de vender un producto solo se requería que este satisficiera una necesidad.

De los años 10 a los 50's había mucha demanda y pocos productos, por lo tanto, el enfoque al servicio no era prioridad, pues había una oferta limitada de empresa que ofrecían un producto y por ende se mantenía la atención de los clientes. En los años 60's comenzaron a cambiar los hábitos de consumo, empezaron a surgir nuevas empresas en el mercado que ofrecían los mismos productos, pero aún sin tener un enfoque en el servicio. Durante los años 70's, la satisfacción de los clientes comenzó a tener una gran importancia, pues la pérdida de clientes por este motivo cada vez se hacía más notoria. La prioridad de las empresas se basaba en producir sin tener en cuenta agregarle a su producto una excelente atención al cliente ofreciendo un servicio de calidad. En la década de los 80 's se comenzó a hablar más sobre calidad en el servicio, satisfacción al cliente y surgieron entidades que ofrecían capacitaciones sobre servicio al cliente. En los años 90's con los avances tecnológicos surgió el concepto de servicio personalizado, individual, privado; generando una experiencia única a los clientes.

Con el surgimiento de las normas ISO se estandarizaron los procesos con el fin de garantizar que las empresas ofrezcan un servicio de excelencia, el cual consiste en diferenciarse de la competencia, que fomente la fidelidad en los clientes, generando una ventaja competitiva y clientes satisfechos. Por lo tanto, hoy en día el servicio al cliente es visto como una estrategia, con la cual se puede lograr diferenciarse de la competencia, creando valor para la empresa y atrayendo cada vez más clientes por ese servicio ofrecido.

A lo largo de la historia, el enfoque de las empresas hacia el servicio ha ido cambiando, debido a que cada día los clientes son más exigentes. En un principio las empresas ofrecían diversos productos los cuales los clientes escogían por su calidad y precio, pero estas dos características llegan a ser fácilmente imitables por la competencia, por lo tanto, se vio la necesidad de incluir un valor agregado con el cual se lograrán diferenciar. 
Los constantes avances tecnológicos han traído consigo un aumento considerable de empresas que ofrecen un mismo producto o servicio, por lo tanto, cada vez es más importantes para las empresas diferenciarse de las demás, con el fin de atraer a los clientes y que estos los prefieran, alcanzado así un alto nivel de competitividad.

El servicio como elemento diferenciador es crucial para que las empresas puedan ser competitivas y perdurar en el tiempo. La competencia es una de las fuerzas más poderosas de la sociedad para avanzar en muchos ámbitos del esfuerzo humano. (Porter, 1998). Las organizaciones de todos los campos y/o sectores tienen que competir para generar y aportar un mayor valor.

Teniendo en cuenta que el tema principal a tratar en el presente proyecto es el servicio, en primera instancia se realizara una revisión de las opiniones de varios autores que han desarrollado el tema, con el fin de encontrar e identificar la naturaleza de los servicios diferenciadores orientados al cliente.

Según Grönroos (1990), una estrategia de servicio, se basa en crear una serie de servicios adicionales para consolidar e incrementar las relaciones con los clientes (pp. 17). De esta forma, no solo le permite a la empresa diferenciar su oferta y crear un valor añadido para sus clientes, sino que también ayuda a mantener a los competidores alejados de las relaciones con sus clientes.

Según Stanton, Etzel y Walker (2007), definen los servicios como actividades identificables separadas e intangibles que son el objeto de una transacción ideada para proveer la satisfacción de un deseo o necesidad (pp. 349). Es por esto que todas las organizaciones deben definir cuáles serán las actividades claves que harán satisfacer las necesidades de los clientes con el fin que los clientes regresen y vuelvan a comprar y puedan recomendarles a los demás consumidores.

Según Kotler, Bloom y Hayes (2004), los servicios se distinguen de los bienes físicos por su carácter intangible, inseparable variable y perecedero. Además, el criterio de satisfacción es diferente y el cliente participa en el proceso debido a que cada experiencia causa una impresión (buena o mala) conocida como el $<<$ momento de la verdad $>>$ (pp. 27). De acuerdo a lo anterior es por esto que el buen servicio al cliente debe estar en todo negocio no solo durante la venta si no también una vez se haya concretado lo que hace que el cliente sienta una experiencia con respecto al servicio prestado, dando retroalimentación que le permitirá a la organización mejorarlas.

Según la American Marketing Association (A.M.A.), los servicios abarcan los elementos intangibles que son inseparabilidad; que implican generalmente la participación del cliente en una cierta manera importante. Por este motivo es necesario conocer la voz del cliente para así poder cumplir sus expectativas y que de esta forma nos prefieran.

Por su parte, Christopher, Payne, \& Ballantyne (2003), definen la calidad del servicio como la habilidad de una organización para satisfacer o superar las expectativas de los clientes, siendo estas los deseos y necesidades que ellos piensan que debería suministrarles la oferta y no lo que podría suministrarles. (pp. 140), es por esto que es importante mantener un alto enfoque en el servicio debido a que representa un valor agregado para la empresa y por ende a los clientes, pues es una estrategia que es difícil de imitar, dado que se basa en incrementar la relación con los clientes a través de ofrecerles un mejor servicio.

El concepto de servicio abarca una gran cantidad de variables que impactan en el crecimiento y sostenibilidad de las empresas, al igual que la percepción de los clientes hacia lo que le ofrecen y lo que esperan recibir. Por lo tanto, acompañado al producto o servicio que adquiere el cliente debe estar sumado un conjunto de características que lo hacen mejor y hace que el cliente nos prefiera en comparación de la competencia.

Para entender el concepto de servicio diferenciador generado a través de un valor agregado para el cliente, se debe apoyar primeramente en el concepto de Marketing acerca del concepto Valor donde establece que "alcanzar los objetivos de la organización depende de la determinación de las necesidades y deseos de su mercado entregando la satisfacción deseada de forma efectiva y eficientemente que los competidores (Kotler y Armstrong, 1988; P14)". Es aquí donde nace la importancia de conseguir que este valor se convierta en un servicio diferenciador donde cada cliente esté dispuesto a pagar el precio que sea sin importar el competi- 
tivo que el mercado tenga en ese momento; dicho valor le permitirá a la organización abarcar nuevos mercados y a mantener la fidelidad y lealtad de sus clientes.

Tal como dice Grönroos (1998), no tiene ningún valor un producto si no tiene un servicio adicional. Por lo tanto, el cliente siempre espera un complemento a ese servicio y/o producto que termina siendo, el servicio y la atención. Es por esto que las empresas deben siempre trabajar en brindarles un valor agregado e innovador en el servicio que presta; donde ellos puedan notar el servicio exclusivo que tienen y puedan sentirse que exceden sus expectativas y su deseo como consumidor; permitiendo que los frentes competidores no absorban su mercado ya cubierto y ganado.

Con relación a lo anterior, Von Hippel (1976) menciona que los clientes pueden desempeñar un papel muy importante para contribuir con la innovación de procesos industriales. Esta es la forma en que los dos ganan, las empresas se motivarán para crear e innovar productos que cubran necesidades y generen satisfacción a los clientes, por medio de procesos y mejora en la calidad de servicio al cliente.

La razón anterior impulsa a la necesidad de identificar los factores que determinan el diseño de un servicio diferenciador con el fin de atraer y mantener sus clientes potenciales, así como lo indi- ca Karl Albrecht (2000), "la estrategia del servicio debe considerarse como un principio organizacional que permita que la compañía trabaje en y para una empresa de servicio, y así canalizar su esfuerzo hacia servicios enfocados en el beneficio que se distingan muy bien ante los ojos del cliente".

Para Matthyssens y Vandenbempt, (1998, p. 347), los nuevos servicios deben desarrollarse en estrecha colaboración con los clientes con el fin de llegar a encajar perfectamente con el mercado, esto quiere relacionar la participación que debe tener el cliente con el desarrollo de la empresa, de los productos y del mejoramiento continuo en la calidad del servicio, siendo esto fundamental para que la empresa tenga clientes felices y puedan posicionarse en el mercado.

\section{Análisis sectorial en empresas del sector de suministros industriales}

A través del análisis sectorial se pretende relacionar a la empresa con su medio y determinar qué aspectos representan a la empresa: fortaleza, debilidades, oportunidades y amenazas.

Teniendo en cuenta lo anterior podemos utilizar el análisis de las 5 fuerzas de Porter, para analizar el entorno específico de aquellos factores que influyen en un grupo específico de empresas que tiene unas características comunes y que concurren en un mismo sector, en este caso para el Sector de Suministro Industriales.

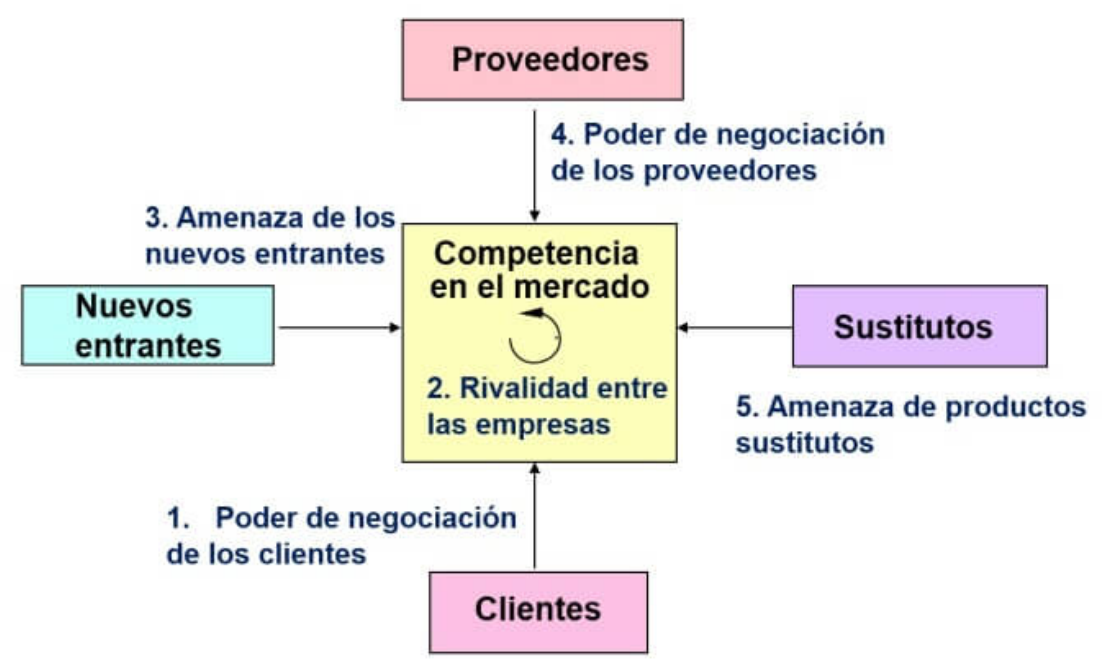

Figura 1. Modelo de las 5 fuerzas de Porter

Fuente: Porter (1998) 
Poder de negociación de los clientes: Para las empresas de servicio del sector de suministros industriales es importante la diversidad de localidades las cuales le facilita el poder ir a comprar a los diferentes puntos de ventas una vez requieran de los productos o servicios que ofrezcan estas empresas para su satisfacción más oportuna y entregas a tiempos, otra de las variables importantes es la calidad y los precios esto es un punto importante y clave para los clientes ya que ellos ven llamativo y atractivo teniendo en cuenta que enmarcan la diferencia frente a la competencia del mismo sector, una vez se tenga en cuenta estas variables se puede captar a los clientes y así se puede lograr tener un servicio diferenciador frente competencia.

Poder de negociación de proveedores: Los proveedores tienen un bajo poder de negociación puesto que el número de proveedores que pueden vender a las empresas del sector de suministro industriales los productos que necesita es alto y estos productos no están diferenciados. Esto beneficia a las empresas de dicho sector debido a que pueden conseguir los productos que necesita a un coste más bajo y a un menor tiempo de entrega (lead time), lo cual favorece para poder cumplirle a tiempo a los clientes.

Rivalidad entre los competidores existentes: Por un lado, existe un número elevado de empresas que ofrecen el mismo servicio de comercialización de equipos y suministros industriales no todos teniendo el mismo portafolio, donde el mayor fuerte de rivalidad entre competidores es la que ofrezca un servicio especializado y/o diferenciador como por ejemplo entregar sus pedidos en el menor tiempo posible, pedidos por app entre otros.

Productos o servicios sustitutivos: Existen empresas que ofrecen otro tipo de comercialización de equipos y suministros industriales diferente de los productos que ofrece empresas de este mismo sector; que pueden ser sustitutivos y que suponen una fuerte competencia como por ejemplo Home Center, Meico, Sumatec, Solmag, Mundial del tornillo entre otros, que venden cada uno un portafolio similar al de Grainger Colombia.

La amenaza de entrada de nuevos competidores: La mayor o menor dificultad para la entrada de nuevos competidores está en función de las barreras de entrada para acceder al sector. Existen, por ejemplo, más barreras para entrar en el sector de Suministro industriales con un portafolio Global que para montar cada uno que contenga parte de dicho portafolio, cuando más fácil sea el acceso de una empresa al sector mayor es la competencia.

En el caso del sector de equipos y suministros industriales, una de las ventajas es que al ser empresas con alta experiencia dominan las tecnologías que hacen referencia a ese sector, y claro está que tienen una mayor facilidad de comprar.

\section{El caso de Grainger Colombia}

Grainger es una multinacional norteamericana líder de suministros industriales, contando con un portafolio de productos de más de 6.000 referencias en tornillería, herramientas manuales y eléctricas, equipos de manipulación de carga, bombas, motores, iluminación y varias marcas propias.

La filial de Grainger en Colombia inició operaciones en el 2010, después de una negociación, entre dos grandes jugadores de suministros industriales, Grainger (ventas de US\$6.200 millones en 2009) y Torhefe (ventas de US\$23 millones en 2009), el cual posee una posición mayoritaria del $80 \%$ en la empresa conjunta, con THF International SAS manteniendo el $20 \%$, esta última con 26 años de experiencia en el mercado colombiano, llega una propuesta a este sector, que ofrecerá productos para el mantenimiento, reparación y operación de pequeñas, medianas y grandes industrias.

Con más de 80 años de historia, la apuesta Grainger por Colombia, se basa en la consecución de un buen socio estratégico como Torhefe y en un mercado en crecimiento, así lo explicó César Lanuza, vicepresidente regional de Grainger para América Latina: "Vemos un enorme potencial en Colombia y creemos firmemente que podemos ayudar para que las compañías del país sean más eficientes

La compañía comenzó a operar con una red de distribución, con presencia en las principales ciudades de Colombia, contando con 5 sucursales $\mathrm{y}$ un centro de distribución ubicadas de manera estratégica, con el fin de ser los más veloces del mercado y atender prontamente a nuestros clientes en sector industrial y ferretero (Comercio). 
"Grainger Colombia reúne la rica historia de servicio al cliente de Torhefe en Colombia y la inigualable cadena de suministro norteamericana de Grainger y su compromiso con el SERVICIO", dijo Court Carruthers, presidente de Grainger International. "Grainger continúa realizando inversiones estratégicas en Latinoamérica que permiten a las empresas tener acceso a una amplia línea de productos y soluciones que ayudan a mantener sus operaciones funcionando de manera eficiente y mantener a sus empleados seguros en el trabajo".

En el 2011, La empresa conjunta tomó la decisión de operar bajo el nombre de marca Torhefe en sus ubicaciones existentes, pasando al nombre de Grainger Colombia; "Compartimos una pasión común por brindar un servicio superior a nuestros clientes", dijo Carlos Acevedo, presidente de Torhefe. "Esperamos una transición sin fisuras para nuestros empleados y clientes y sabemos que ellos apreciarán y se beneficiarán de la amplia oferta y disponibilidad de productos que viene de unir fuerzas con el principal distribuidor industrial de línea ancha de Norteamérica".

A finales del año 2012, los socios de Grainger tomaron la decisión de adquirir el $20 \%$ restante que tenía la empresa familiar Torhefe, con el fin de adquirir el $100 \%$ de totalidad de esta empresa.

En este mismo periodo define como estrategia ser el proveedor de MRO \#1 en Colombia, para ello definió planes que permitieran dar a los clientes la mejor experiencia de servicio comparado con los demás competidores.

Entre los años 2014 hasta 2015, Grainger Colombia decide abrir un centro de distribución con más del $8000 \mathrm{mt} 2$ con acceso a más de un millón de productos en los EEUU.

Durante el 2016, Grainger trabajó en la identificación de su TOP de clientes potenciales para la sucursal de Barranquilla y Cartagena con el fin de desarrollar una estrategia que permita agregarle valor al servicio presentado a este tipo de clientes calificados que permitan mantenerlo y atraerlos por el mismo costo pagado, su mayor foco estratégico es el Servicio a sus clientes.

Actualmente Grainger es un distribuidor interempresarial, el cual cuenta con una oferta de pro- ductos disponibles de más de 35 mil referencias en inventario, los cuales son necesarios para mantener, reparar y operar cualquier instalación, logrando así una distribución de productos y soluciones acorde con las necesidades del mercado, apoyando a la Industria nacional para que su operación nunca se detenga.

La visión actual de Grainger es: "Ser el proveedor de MRO \#1 en Colombia, para ello trabajamos en dar a nuestros clientes la mejor experiencia, que el recibo de su orden sea tiempo y completa, lo hacemos cuidando del inventario de Grainger, y manteniéndolo en óptimas condiciones, exactitud y control".

Así mismo se mide la gestión, con el fin de sorprender al cliente y perseguir los mejores resultados.

Hoy en día caracterizar a los clientes no es más que segmentar, dividir o clasificar clientes en grupos que tengan características, deseos y posibilidades semejantes, Grainger Colombia cuenta con dos tipos de segmento en su maestro de cliente, los cuales son clientes industriales y comerciales en cada una de sus sucursales. Actualmente su mayor $\%$ de ventas y el mayor número de clientes potenciales lo tiene representado la sucursal de Barranquilla y Cartagena.

\section{Metodología}

Esta investigación es de tipo cuantitativo, la cual permitió conocer la relación que existe entre el core del negocio y la percepción de los diferentes grupos de interés, tales como Clientes Internos, Externos, Proveedores y Accionistas con respecto al servicio ofrecido.

Para la recopilación de datos de esta investigación se acudió al empleo de encuestas para medir las expectativas del cliente, con el propósito de identificar cuál es el elemento diferenciador que el cliente espera al momento de obtener el servicio, que sea determinante en la decisión de compra y que la competencia no lo tenga. Con ello se pretende conocer cuáles son las necesidades básicas y requerimientos relacionados con el concepto y promesa de valor de la empresa que permitan definir cuál será el servicio diferenciador para sus clientes y su ventaja competitiva 
en el mercado, así los resultados de investigación se apoyan en técnicas de investigación válidas en el medio.

- Fase 1: Se identificó el segmento a investigar, con el fin de definir la muestra de los diferentes actores a los cuales se les aplicó el instrumento, con una muestra de 158 clientes para una población de 200 , con lo cual se obtuvo un margen de error del $3.6 \%$, con intervalo de confianza utilizado de $95 \%$ y una heterogeneidad del $50 \%$.

- Fase 2: Se realizó un análisis cuantitativo de los resultados de las encuestas, realizando en primera instancia una prueba piloto para validar el instrumento a través del alfa de Crombach.

- Fase 3: Se consolidó la información en tablas y gráficos, de tal forma que permita observar los resultados.

- Fase 4: Se realizó un análisis factorial con el fin de identificar los factores claves que inciden en la satisfacción del cliente.
- Fase 5: Con los resultados obtenidos se propone un diseño de servicio diferenciador para empresas del sector de suministros industriales.

\section{Resultados}

Para la aplicación de la prueba piloto, se realizó una selección de 50 clientes del segmento industria, con el fin de validar el instrumento a utilizar, esta aplicación de la encuesta piloto fue realizada a través de correo electrónico dándole un especial seguimiento al cumplimiento de las respuestas de dicha encuesta, las preguntas de la encuesta van enfocadas a cuales son las necesidades y expectativas que los clientes esperan de empresas que sean del sector de suministros industriales obtenidos a través de la empresa Grainger para lograr ser atractivos y tener un servicio diferenciador frente a los demás competidores.

Tal como se puede observar en la fig. 2. Del resultado obtenido de la prueba piloto, una de las necesidades básicas más importantes para los clientes radica en que el $40 \%$ de los 50 clientes tomados para la prueba piloto, solicitan que se cuente con un servicio diferenciador que permitan capturarlos y atraerlos como clientes potenciales.

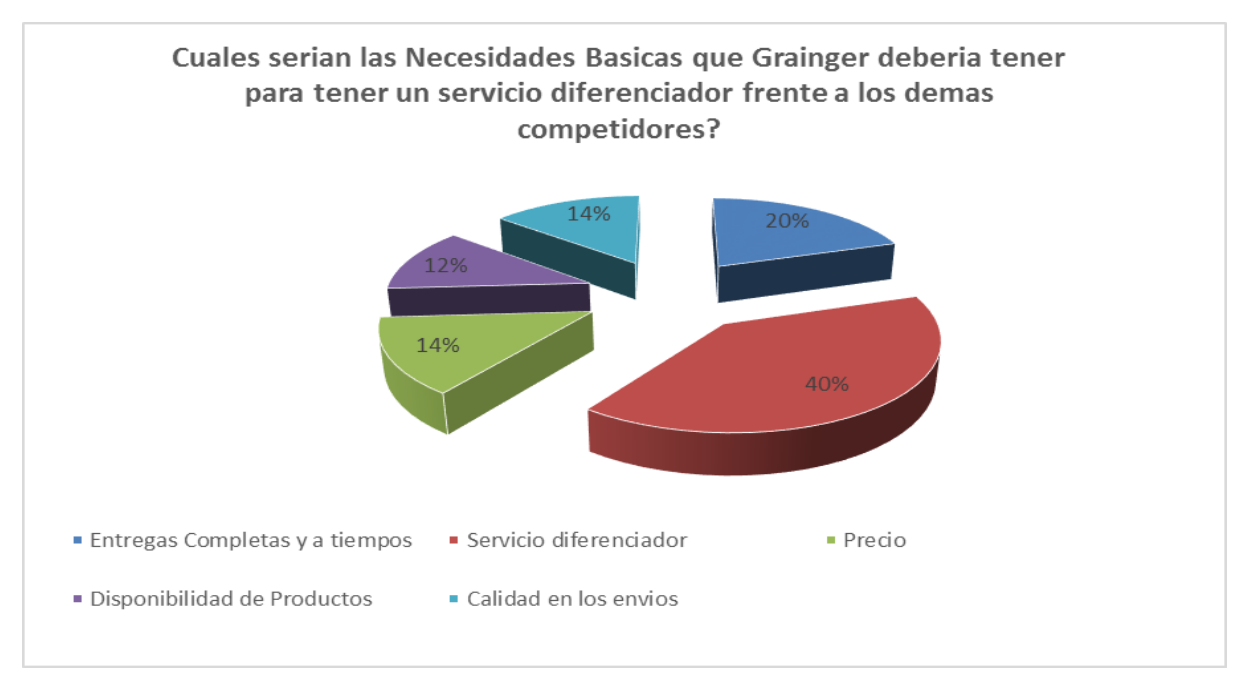

Figura 2. Resultado $1^{\mathrm{a}}$ pregunta prueba piloto

Fuente: elaboración de los autores

Así mismo observamos en la fig.3, donde el $30 \%$ de los 50 clientes encuestados indican que uno de los requerimientos fundamentales para este tipo de segmento es contar con una atención especializada donde ellos puedan sentirse más atraídos por querer consumir los productos de Grainger Colombia. 
Factores que Determinan el Diseño de Servicios Diferenciadores en Empresas del Sector de Suministros Industriales: Caso GRAINGER

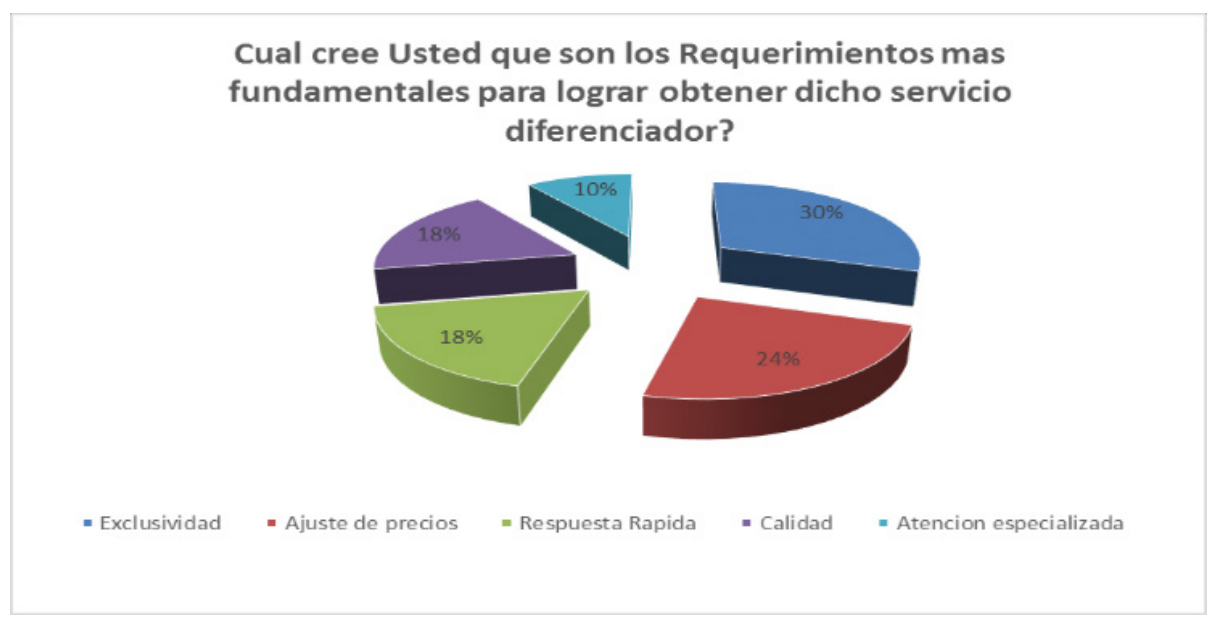

Figura 3. Resultado $2^{\mathrm{a}}$ pregunta prueba piloto

Fuente: elaboración de los autores

Una vez que ellos puedan contar con ese servicio diferenciador y este cuente con una atención especializada, podemos observar que para los clientes de acuerdo a los resultados obtenidos tal como indica la fig.4, lo más importante es que se mantengan los precios, con una entrega efectiva y una excelente atención a clientes.

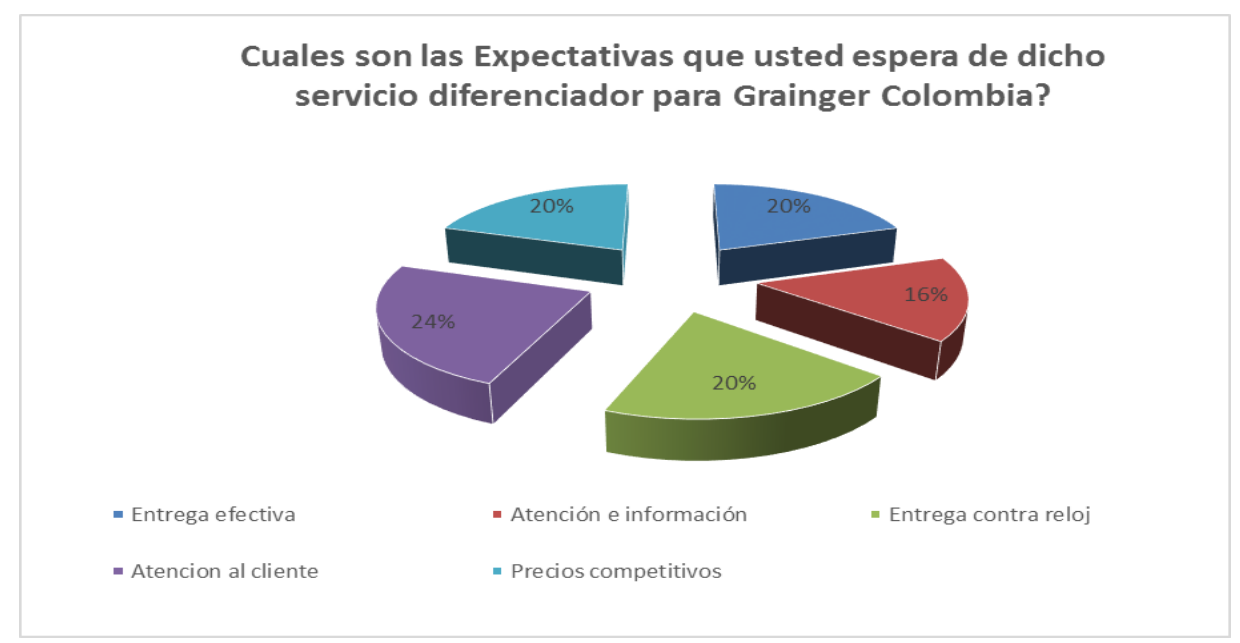

Figura 4. Resultado $3^{\mathrm{a}}$ pregunta prueba piloto

Fuente: elaboración de los autores

\section{Análisis de percepción del grupo de interés}

En la encuesta se evaluó la percepción que tienen los clientes del sector de suministros industriales, específicamente en el segmento Industria, con respecto al servicio que reciben de parte de la empresa Grainger.

Se analizaron puntos importantes como son el tiempo de entrega, estado de recepción del pedido, atención de servicio post venta, entre otros. La encuesta se aplicó a una muestra representativa de clientes del segmento industria de la empresa Grainger, aplicando en total 158 encuestas a clientes escogidos de manera aleatoria de la base de datos de la empresa Grainger.

Con el fin de validar la fiabilidad de los ítems que componen el instrumento, se aplicó Alfa de Cronbach al total de la muestra en estudio, con un total de 8 ítems, dando como resultado 0.868 , indicando que el instrumento es altamente fiable, siendo que el valor mínimo aceptable para alfa $>0.7$. La prueba de KMO y Barlett de adecuación de muestreo mostró un resultado de 0,847 mientras que la prueba de esfericidad mostró los valores anotados en la tabla 2. 
Tabla 2. Prueba de KMO y Barlett

\begin{tabular}{lrr}
\hline $\begin{array}{l}\text { Medida Kaiser-Meyer-Olkin de adecuación de } \\
\text { muestreo }\end{array}$ & 0,847 \\
Prueba de esfericidad de Bartlett & Aprox. Chi- & \\
& cuadrado & 8132,798 \\
& Sig. & 190 \\
& Sig. &, 000 \\
\hline
\end{tabular}

Fuente: elaboración de los autores a partir de SPSS

Una vez validada la fiabilidad de los datos obtenidos en las encuestas, se procedió a realizar la consolidación y análisis de los mismos, con el fin de conocer cuál es la opinión de los clientes del sector de suministros industriales sobre el servicio recibido, y a su vez poder identificar cual sería el servicio diferenciador adecuado que las empresas de este sector deberían aplicar para que represente un valor agregado para los clientes.
Tal como se muestra en la Tabla 3, se logró evidenciar que las causales que más representan la insatisfacción de los clientes se deben a que el $50 \%$ ocurre por incumplimiento en la hora de entrega de los pedidos y el $39 \%$ por falta de disponibilidad de mercancía inmediata.

Tabla 3. Datos de resultado de encuesta de servicio de clientes

\begin{tabular}{lcc}
\hline \multicolumn{2}{c}{ Cuál es la principal causa para no considerar a Grainger como una opción } \\
\hline Answer Options & Response Percent & Response Count \\
Poca Disponibilidad & $39 \%$ & 63 \\
Demora en la entrega & $50 \%$ & 79 \\
Mal servicio por parte del asesor & $19 \%$ & 30 \\
Errores en despacho & $20 \%$ & 32 \\
Errores en las cotizaciones & $5,7 \%$ & 9 \\
Falta de soporte y servicio post venta. & $22 \%$ & 36 \\
answered question & & 158 \\
skipped question & & 279
\end{tabular}

Fuente: elaboración de los autores

Debido a lo anterior se hace necesario el ta mejorar el servicio prestado y de igual forma diseño de un servicio diferenciador que permi- atraer nuevos clientes y mantenerlos.

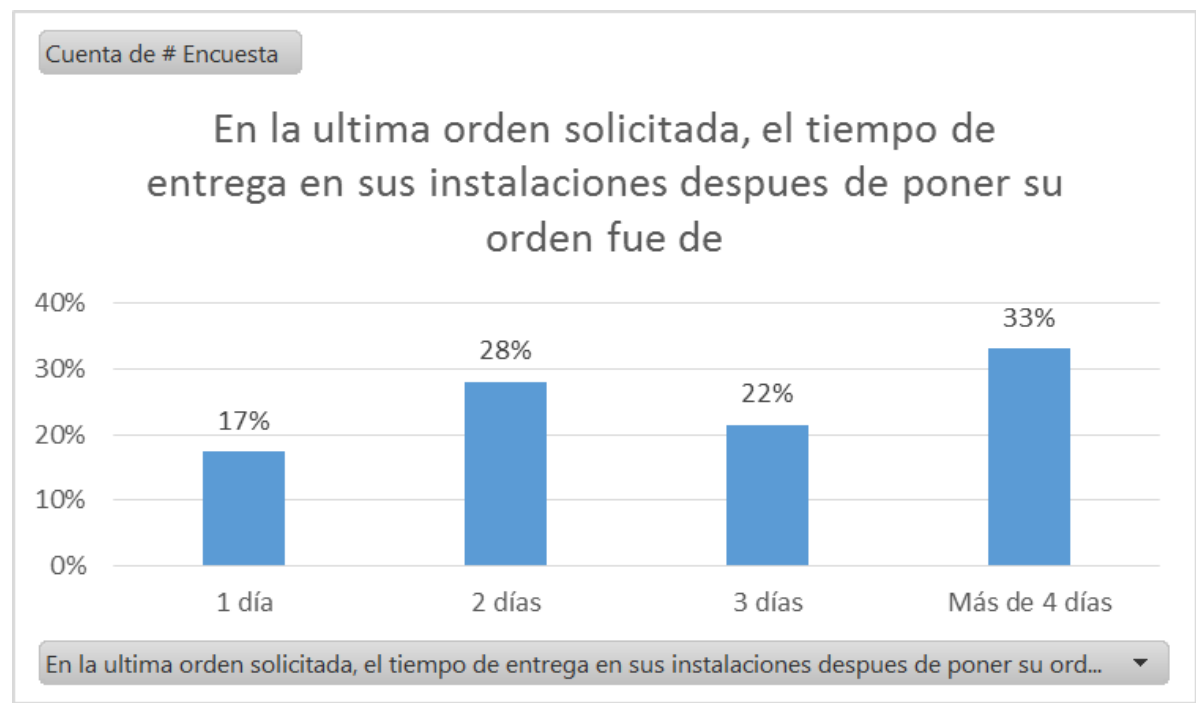

Figura 5. Percepción de los clientes sobre tiempos de entrega

Fuente: elaboración de los autores 
Factores que Determinan el Diseño de Servicios Diferenciadores en Empresas del Sector de Suministros Industriales: Caso GRAINGER

En la fig. 5, se puede apreciar que el 33\% de los encuestados afirman que reciben sus órdenes en más de 4 días, siendo este un punto desfavorable, ya que los clientes requieren que sus pedidos sean entregados en el menor tiempo posible.

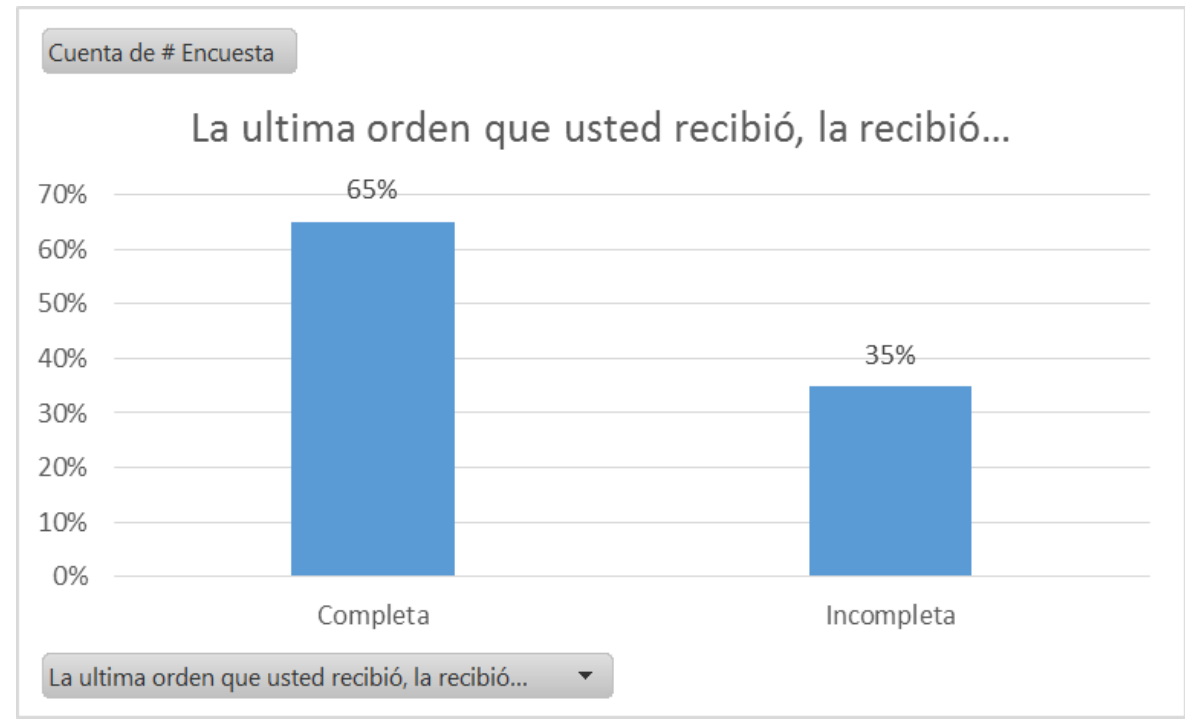

Figura 6. Percepción de los clientes sobre el estado de la orden

Fuente: elaboración de los autores

La fig. 6 nos muestra que el $65 \%$ de los cli- siendo este uno de los aspectos más relevantes entes manifiestan recibir sus pedidos comple- para la empresa a la hora de medir la satisfactos, pero aun así hay un porcentaje significativo ción de los clientes, es importante mejorar este que dice que recibe sus órdenes incompletas, indicador.

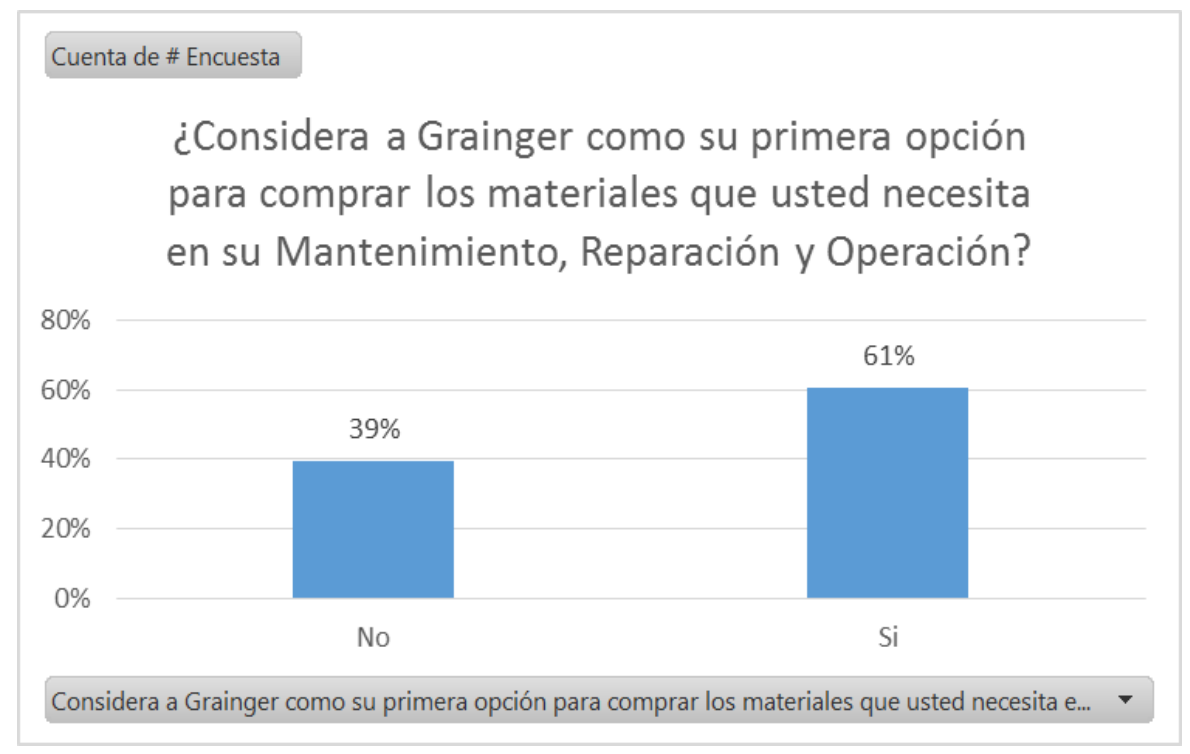

Figura 7. Percepción de los clientes sobre la empresa como primera opción

Fuente: elaboración de los autores

La fig. 7 muestra que el $61 \%$ de los clientes a la hora de comprar materiales de suministros considera a la empresa como su primera opción industriales. 


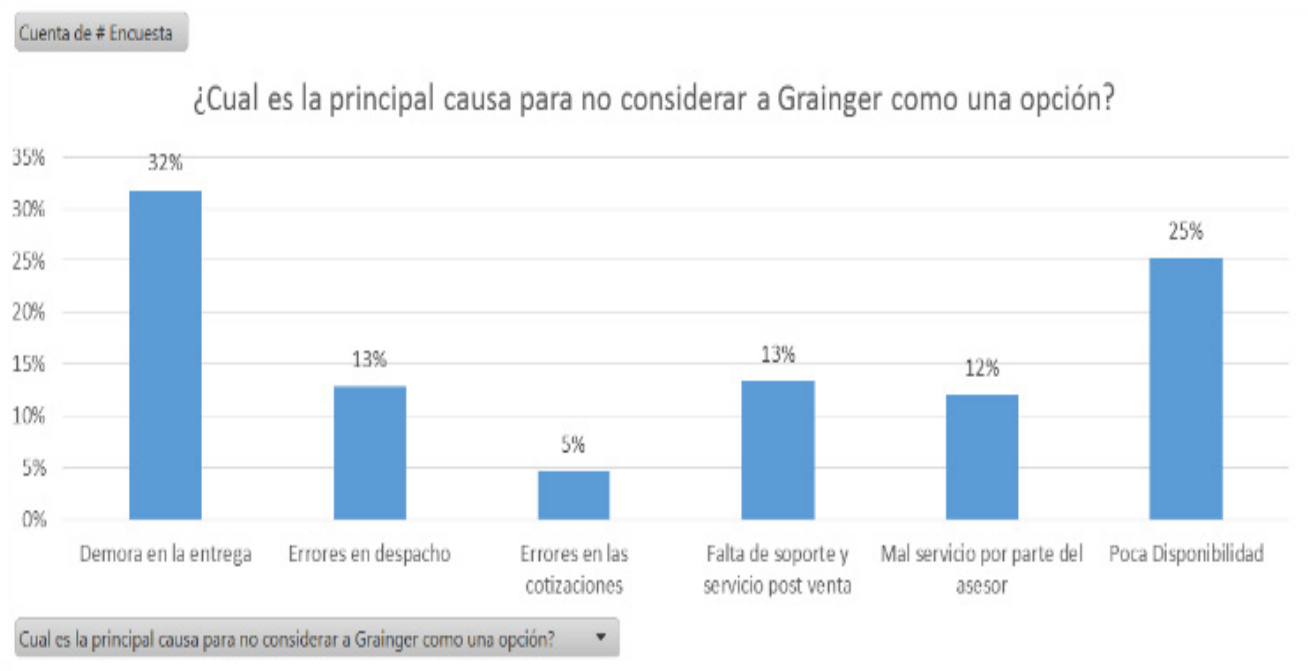

Figura 8. Motivos para no escoger a la empresa como primera opción

Fuente: elaboración de los autores

En la fig. 8 se evidencia que el principal demora en la entrega de sus pedidos, seguido motivo por el cual los clientes no consideran por poca disponibilidad, errores en el despacho a la empresa como su primera opción es por la y falta de soporte y servicio post venta.

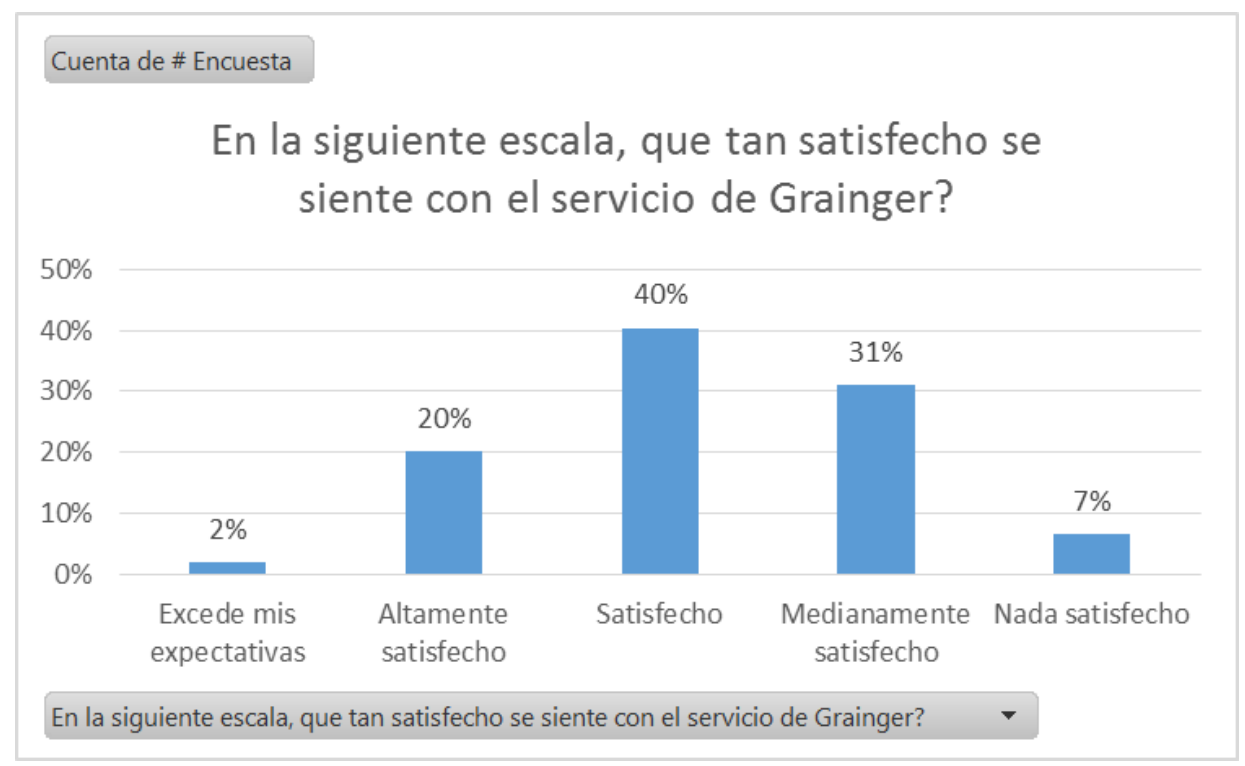

Figura 9. Satisfacción sobre el servicio ofrecido

Fuente: elaboración de los autores

En la fig. 9 se observa que el $40 \%$ de los clientes se sienten satisfechos con el servicio ofrecido y el $31 \%$ se siente medianamente satisfecho, llama la atención que solo el $2 \%$ considera que el servicio excede sus expectativas.

\section{Análisis factorial para identificar los facto- res que más inciden en la satisfacción de los clientes}

Una vez consolidados los datos de las encuestas, se procedió a realizar el análisis factorial a través del uso del Software SPSS Statistics. En la tabla 4 se muestran los cinco (5) componentes, los cuales explican el 78\% de la varianza, siendo estos los más relevantes para la satisfacción de los clientes. 
Factores que Determinan el Diseño de Servicios Diferenciadores en Empresas del Sector de Suministros Industriales: Caso GRAINGER

Tabla 4. Varianza total explicada

\begin{tabular}{|c|c|c|c|c|c|c|c|c|c|}
\hline \multicolumn{10}{|c|}{ Varianza total explicada } \\
\hline & \multicolumn{3}{|c|}{ Autovalores iniciales } & \multicolumn{3}{|c|}{$\begin{array}{c}\text { Sumas de cargas al cuadrado de } \\
\text { la extracción }\end{array}$} & \multicolumn{3}{|c|}{$\begin{array}{c}\text { Sumas de cargas al cuadrado de } \\
\text { la rotación }\end{array}$} \\
\hline & Total & $\begin{array}{c}\% \text { de } \\
\text { varian- } \\
\text { za }\end{array}$ & $\begin{array}{l}\% \text { acumu- } \\
\text { lado }\end{array}$ & Total & $\begin{array}{l}\% \text { de va- } \\
\text { rianza }\end{array}$ & $\begin{array}{l}\% \text { acumu- } \\
\text { lado }\end{array}$ & Total & $\begin{array}{l}\% \text { de va- } \\
\text { rianza }\end{array}$ & $\begin{array}{l}\% \text { acumu- } \\
\text { lado }\end{array}$ \\
\hline 1 & 8,821 & 44,105 & 44,105 & 9,821 & 44,105 & 44,105 & 8,623 & 43,113 & 43,113 \\
\hline 2 & 2,599 & 12,993 & 57,098 & 2,599 & 12,993 & 57,098 & 1,953 & 9,763 & 52,875 \\
\hline 3 & 1,864 & 9,322 & 66,420 & 1,864 & 9,322 & 66,420 & 1,924 & 9,621 & 62,496 \\
\hline 4 & 1,417 & 7,085 & 73,504 & 1,417 & 7,085 & 73,504 & 1,732 & 8,662 & 71,159 \\
\hline 5 & 1,044 & 5,218 & 79,722 & 1,044 & 5,218 & 78,722 & 1,513 & 7,563 & 78,722 \\
\hline 6 & 0,892 & 4,461 & 83,183 & & & & & & \\
\hline 7 & 0,748 & 3,738 & 86,921 & & & & & & \\
\hline 8 & 0,503 & 2,513 & 89,434 & & & & & & \\
\hline 9 & 0,432 & 2,159 & 91,593 & & & & & & \\
\hline 10 & 0,411 & 2,056 & 93,649 & & & & & & \\
\hline 11 & 0,275 & 1,375 & 95,025 & & & & & & \\
\hline 12 & 0,252 & 1,259 & 96,283 & & & & & & \\
\hline 13 & 0,208 & 1,038 & 97,322 & & & & & & \\
\hline 14 & 0,163 & 0,816 & 98,138 & & & & & & \\
\hline 15 & 0,103 & 0,513 & 98,650 & & & & & & \\
\hline 16 & 0,074 & 0,369 & 99,019 & & & & & & \\
\hline 17 & 0,064 & 0,322 & 99,341 & & & & & & \\
\hline 18 & 0,053 & 0,264 & 99,605 & & & & & & \\
\hline 19 &, 050 & 0,249 & 99,854 & & & & & & \\
\hline 20 & 0,029 & 0,146 & 100,000 & & & & & & \\
\hline
\end{tabular}

Método de extracción: análisis de componentes principales.

Fuente: elaboración de los autores

La Tabla 5 muestra la matriz de componentes rotados, en la cual se puede apreciar la correlación entre cada variable y el factor establecido por el programa. Estos valores indican el nivel de relevancia de la variable para cada factor, y de esta forma se determina la asignación que se le debe dar basándose en la carga factorial suministrada por el modelo.

Se puede observar que el primer factor es el que más variables tiene correlacionadas, ya que cuenta con un total de 12 variables, el segundo factor cuenta con 3 variables, el tercer factor con 2 variables, el cuarto factor cuenta con 1 variable, y por último el quinto factor cuenta con 2 variables correlacionadas. En la tabla 6 se puede observar el detalle de la agrupación de cada factor.

Una vez agrupadas las variables en cada factor correspondiente, se procede a renombrar cada uno de los factores, basándose en la similitud que hay entre las variables. 
Tabla 5. Matriz de componente rotado

\begin{tabular}{|c|c|c|c|c|c|}
\hline & \multicolumn{5}{|c|}{ Componente } \\
\hline & 1 & 2 & 3 & 4 & 5 \\
\hline Nivel de satisfacción con el servicio ofrecido & 0,935 & & & & \\
\hline Tiempo de entrega de su pedido después de montada la orden & 0,914 & & & & \\
\hline Valor agregado & 0,907 & & & 0,232 & \\
\hline Estado en el que recibió su orden & 0,902 & & $-0,308$ & & \\
\hline Considera a Grainger su primera opción & 0,901 & & $-0,229$ & & \\
\hline Instructivo de fácil acceso & 0,889 & 0,211 & & & \\
\hline Catalogo virtual & 0,883 & & & 0,266 & \\
\hline Atención personalizada & 0,831 & & & & \\
\hline Respuesta inmediata & 0,83 & & & 0,207 & \\
\hline Exclusividad en el servicio & 0,61 & 0,385 & $-0,221$ & 0,513 & \\
\hline Atención de acuerdo a sus necesidades & 0,575 & $-0,4$ & & 0,341 & \\
\hline Poca disponibilidad & 0,554 & & $-0,393$ & & $-0,312$ \\
\hline Falta de soporte y servicio post venta & & 0,893 & & & \\
\hline Mal servicio por parte del asesor & 0,409 & $-0,599$ & 0,412 & $-0,329$ & \\
\hline Errores en despacho & 0,482 & 0,564 & & & \\
\hline Errores en las cotizaciones & & & 0,881 & & \\
\hline Precios competitivos & & & 0,679 & 0,275 & 0,224 \\
\hline Seguimiento a ordenes & & & & 0,852 & \\
\hline Calidad en el servicio al cliente & & & & 0,263 & 0,825 \\
\hline Demora en la entrega & 0,272 & & & $-0,36$ & 0,706 \\
\hline
\end{tabular}

Método de extracción: análisis de componentes principales.

Método de rotación: Varimax con normalización Kaiser.

a. La rotación ha convergido en 7 iteraciones

Fuente: elaboración de los autores

Tabla 6. Variables asociadas a cada factor

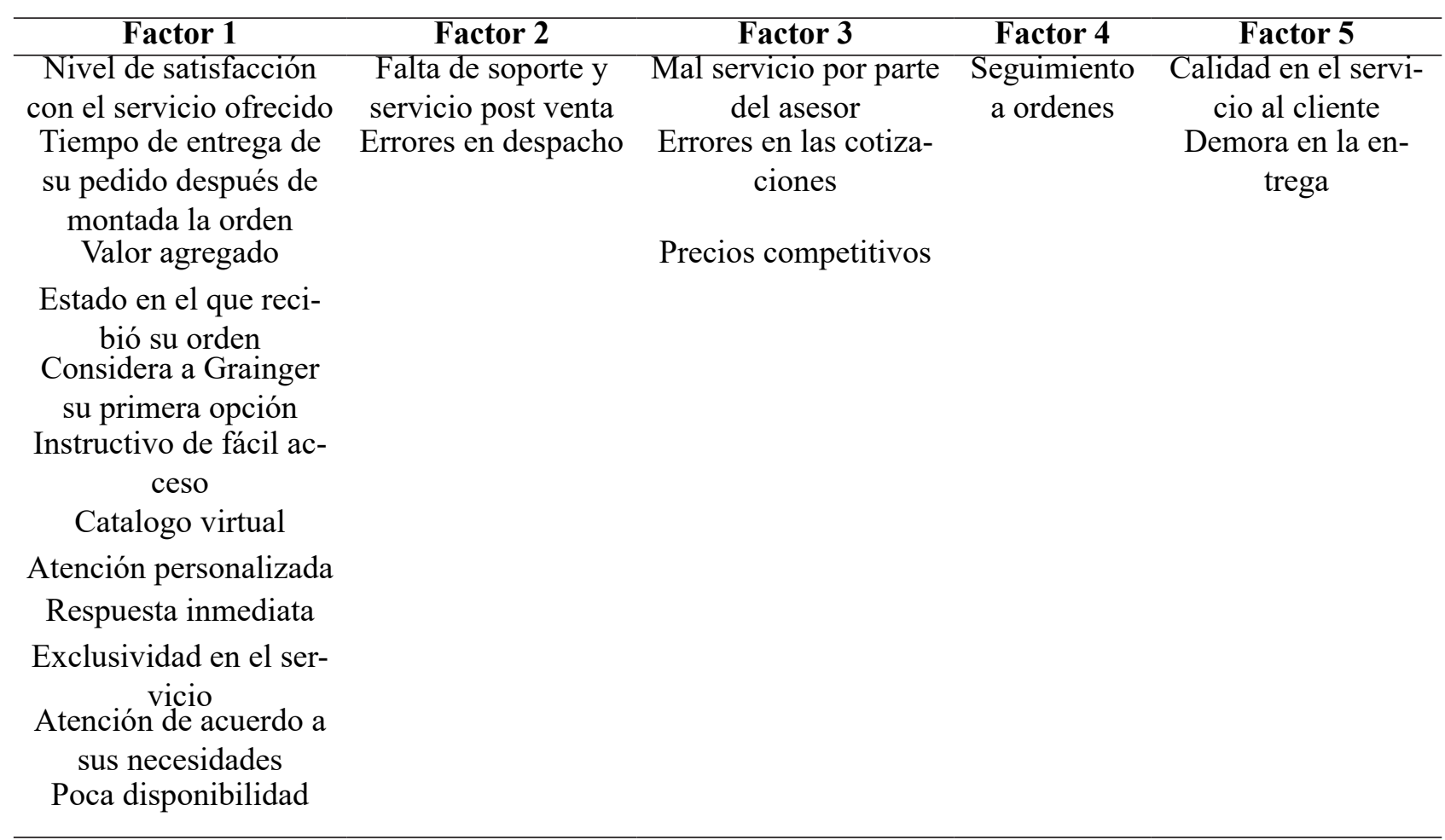

Fuente: elaboración de los autores 
Factores que Determinan el Diseño de Servicios Diferenciadores en Empresas del Sector de Suministros Industriales: Caso GRAINGER

Para cada uno de los factores establecidos, se logró capturar el problema que percibe el cliente, y de esta forma poder plantear una solución y tomar de ahí las bases fundamental- es para el desarrollo de la estrategia que enmarcara cada una de las variables evaluadas por los clientes, como se muestra en la tabla 7.

Tabla 7. Renombramiento de los factores.

\begin{tabular}{|c|c|c|}
\hline Factor & Variables & Renombramiento \\
\hline \multirow{10}{*}{ Factor 1} & Nivel de satisfacción con el servicio ofrecido & \multirow{10}{*}{$\begin{array}{l}\text { Información rápida y a la } \\
\text { mano }\end{array}$} \\
\hline & $\begin{array}{l}\text { Tiempo de entrega de su pedido después de montada la } \\
\text { orden } \\
\text { Valor agregado }\end{array}$ & \\
\hline & Estado en el que recibió su orden & \\
\hline & Considera a Grainger su primera opción & \\
\hline & Instructivo de fácil acceso & \\
\hline & Catalogo virtual & \\
\hline & Atención personalizada & \\
\hline & Respuesta inmediata & \\
\hline & Exclusividad en el servicio & \\
\hline & Atención de acuerdo a sus necesidades & \\
\hline \multirow[t]{2}{*}{ Factor 2} & $\begin{array}{l}\text { Falta de soporte y servicio post venta } \\
\text { Errores en despacho }\end{array}$ & \multirow[t]{2}{*}{ Calidad en el proceso } \\
\hline & Mal servicio por parte del asesor & \\
\hline Factor 3 & $\begin{array}{l}\text { Errores en las cotizaciones } \\
\text { Precios competitivos }\end{array}$ & Atención personalizada \\
\hline \multirow{2}{*}{ Factor 4} & Seguimiento a ordenes & \multirow{2}{*}{ Seguimiento a ordenes } \\
\hline & Calidad en el servicio al cliente & \\
\hline Factor 5 & Demora en la entrega & Entregas efectivas \\
\hline
\end{tabular}

Fuente: elaboración de los autores

Propuesta de diseño de servicio diferenciador para empresas del sector de suministros industriales

La percepción general de los clientes es que las empresas de equipos \& suministros industriales no cuentan con un sistema de información que le permita al cliente tener acceso rápido para consultar y verificar precios, descuentos, disponibilidad de productos, tiempo de entrega, y exclusividad para aquellos pedidos urgentes que lo requieran de manera inmediata.

Para darle solución a los requerimientos de los clientes, se requiere de un servicio que agregue valor, en donde se tenga la información de entrega en tiempo real, muestre un análisis de rotación de productos, que ofrezca un servicio posventa y que proporcione un programa de clientes exclusivos, convirtiéndose en un elemento diferenciador; Es por esto que dichas empresas, deberán desarrollar una plataforma virtual donde el cliente pueda adquirir sus pedidos en el menor tiempo posible y que exista un servicio express donde ellos no tengan que acercarse a las ubicaciones físicas de la empresa; esto conllevará a crear estrategias que sean enfocadas a atraer clientes.

Esta estrategia que se implemente debe llevar a la organización a cumplir su misión de ser la primera opción para sus clientes al momento de requerir o necesitar un servicio, lo que permitirá a la organización tenerlo como el escalón principal para dar el mejor beneficio que se distingan frente a los ojos de los clientes, lo que conlleva a que se tenga mayor acogida en el mercado y la empresa obtenga altas ventas.

\section{Conclusiones y discusión}

Hoy en día las empresas deben centrar su atención en conocer y escuchar la Voz del cliente, con el fin de conocer más a fondo las expectati- 
vas por la que deben trabajar para así alcanzar su mayor objetivo que es atraer a sus clientes con un servicio diferenciador que lo impacte, a esto se le debe incorporar estrategias que vayan encaminadas a generar dicho valor. Teniendo en cuenta lo anterior conocer al cliente le permitirá ofrecerles un servicio acorde a sus expectativas; cabe resaltar que el cliente es la razón primordial de una compañía, es por esto que se debe trabajar continuamente para satisfacer sus deseos y necesidades, la buena relación con los clientes le permite conocer a la compañía cambios y adaptarse a ellos con la finalidad de siempre ir adelante y responder a sus necesidades.

El servicio siempre debe ser un aspecto importante en las organizaciones, tal como lo menciona Albretch (2000), "El servicio es hoy negocio de negocios y la capacidad de servir a los clientes efectiva y eficientemente es un problema que toda la organización debe afrontar", es por ello que se convierte en la base principal para alcanzar el may- or escalón ya que el servicio es uno de los factores de diferenciación en las organizaciones.

Es de conocimiento común que todos los clientes al solicitar un servicio lo quieren a el menor tiempo posible, sin importarle las falencias que en el momento la compañía pueda tener, a él solo le importa que lo atiendan y le den una respuesta rápida es por ello que se hace importante trabajar en metodologías que reduzcan el mayor tiempo perdido en la cadena de suministro con el fin de cumplir con una de las variables críticas que los clientes hoy en día lo exigen y es importante para mantenerlos y fidelizarlos.

Son muy pocas las empresas que trabajan por precios competitivos, muchas tienen precios más altos en el mercado, pero tiene un servicio que los diferencia agregándole algún valor que los demás y los clientes estén dispuestos a pagar por ellos, es decir muchos compran sus productos / servicio por lo que hacen y no por lo que cuesta.

\section{Referencias}

Albrecht, K., \& Zemke, R. (2000). Gerencia del Servicio: Cómo hacer negocios en la Nueva Economía. Bogotá: 3R Editores.

Al-Dweeri, R. M. (2011). La Calidad en los Servicios Electrónicos como Estrategia Competitiva. Modelo de Análisis de sus Componentes y Efectos sobre la Satisfacción y la Lealtad (Tesis Doctoral). Málaga: Servicio de Publicaciones de la Universidad de Málaga.

Armario, Barroso, Ruiz (2004). Valor percibido de un servicio. Revista española de investigación de marketing (8). https://dialnet.unirioja.es/buscar/documentos?querysDismax.DOCUMEN$\mathrm{TAL} \_\mathrm{TODO}=$ desarrollar + estrategias + de + servicio\&inicio $=21$.

Barlow, J., Maul, D., \& Edwarson, M. (2000). Valor Emocional: Creando lazos fuertes con sus clientes. San Francisco: Berrett-Koehler.

Bolton, R., \& Drew, J. (1991). A Longitudinal Analysis of the impact of service changes on customer attitudes. Journal of Marketing 55, 1-9.

Braidot, N. (2006). Neuromarketing: Neuroeconomía y Negocios. Nestor Braidot.

Fleming, J., Coffman, C., \& Harter, J. (Jul-Ago 1995). Manage Your Human Sigma. Harvard Business Review, 107-114.

Frei, F. \&. (2012). Uncommon Service. Boston Massachusetts: Harvard Business Review Press.

Grönroos, C. (1990). Service Management and Marketing: managing the moments of truth in service competition. In DC Heath: Lexington Books. https://doi.org/10.1108/EUM0000000004874 
Factores que Determinan el Diseño de Servicios Diferenciadores en Empresas del Sector de Suministros Industriales: Caso GRAINGER

Hallowell, R. (1996). The relationships of customer satisfaction, customer loyalty and profitability: an empirical study. International Journal of Service Industry Management, Vol. 7 No. 4, 27-42.

Jojn R. Tauser and Kenneth J, W. (1982). Dynamic Analysis of Consumer Response to marketing strategies. management science Vol 28, No 5, 455-486.

Kotler, Bloom, Hayes. (2002). Marketing Professional Services: Forward-Thinking Strategies for Boosting Your Business, Your Image, and Your Profits, 2nd Edition. Consulting to Management, 13(3), 57-58. http://library.capella.edu/login?url=http://search.proquest.com/docview/215900709?accountid=27965

Kotler, P., Cámara, D., Grande, I., \& Cruz, I. (2000). Dirección de Marketing. Madrid: Pretince Hall.

Lovelock C, Reynoso J, D’andrea G, y Huete L. (2004). Administración de Servicios. Estrategias de Marketing de Operaciones y Recursos Humanos. Primera Edición. Pearson Educación, S.A. México.

Lusch, R. \&. (2006). The Service-dominant Logic of Marketing: Dialog, Debate, and Directions. New York: M.E. Sharpe, Inc.

Ma, C., Wang, Z., Xu, X., \& Wu, Q. (2013, 6). Measuring Service Value Based on Service Semantics. Journal of Service Science and Management, 56-68.

Medina Agustín (2010). Bye, Bye, Marketing: Del poder del mercado al poder del consumidor. Editorial Piramide. ISBN 9788436823868.

Porter, M. (1998). Competitive Strategy: Techniques for Analyzing industries and competitors. Glencoe, Il: Free Press.

Porter, M. E. (1998). On competition / Michael E. Porter. Antitrust Bulletin (Vol. 44). http://lcweb. loc.gov/catdir/toc/98007643.html

Robledo, J. (2010) “La Mercadotecnia en las organizaciones: La Gestión del conocimiento en la Mercadotecnia”, Pp. 19-22, Ediciones ILCSA S.A, Primera edición. Universidad Autónoma de Baja California. ISBN 9786077736301

Robledo, J., Del Rio, J., Ruiz, G., Martinez, O. (2015) “Gestión del Conocimiento Organizacional, Fundamentos Teóricos”. Jorale editores y Universidad Autónoma de Baja California (México). ISBN 9786077522249.

Stanton, Etzel y Walker (2007). Fundamentos de Marketing. Decimocuarta Edición. pp. 349. Editorial McGraw-Hill/Interaericana. 